\title{
Celle conveltive e variazioni periodiche del gradiente verticale del campo elettro-atmosferico
}

\author{
(Convective cells and periodic variations of the certical gradient \\ of electro-atmospheric field)
}

R. JANENELLI (*)

Ricevuto il 22 Marzo 1969

\begin{abstract}
Rassunto. -.. Dopo aver descritto brevemente eome si possono oftenere in laboratorio delle celle convettive in mu liquido e nell'aria, si applicano le ricerche di laboratorio alla formazione delle celle convettive nellatmosfera. Siccome qualche apparechio, specialmente lelettrometro. descrive delle variazioni periodiche con periodo eguale alla durata di una cella convettiva. si conclude che tali variazioni si debbano attribuire alla formazione a al dissolvimento di celle convettive nell'atmosfera.
\end{abstract}

Sunmary. - After having briefly described how one can obtain some convective ceels in a liquid and in the air through laboratory experiments, one applies it to the formation of convective cells in the atmosphere. Since certain apparatus, particularly the electrometer, describes some periodic variations, with the period egual to the duration of a convective cell, one can conclude that such variations are to be attributed to the formation and dissolution of convective cells in the atmosphere.

I movimenti, che avvengono in strati instabili di un fluido, sono stati studiati e descritti indipendentemente da molti ricercatori. Ia scoperta della convezione cellulare, che avviene in un fluido instabile, ordinariamente viene attribuita ad Enrico Bénard, il quale descrisse il fenomeno nei suoi minimi particolari.

Molti antori chiamano "cella di Bénard" la cella tipica di convezione, che ha luogo in uno strato di fluido inizialmente in quiete.

$\left({ }^{*}\right)$ Osservatorio Meteorologieo dell'Istituto Cavanis di Venezia. 
Però, per amor del vero, le osservazioni di Bénard furono anticipate di circa 20 anni da James Thompson (fratello di Lord Kelvin), il quale osservò una struttura cellulare (simile ad un mosaico) nell'acqua saponata, che si raffreddava all'uscita di un tubo. In seguito Thompson riprodusse il fenomeno in laboratorio.

Le celle di convezione si possono ottenere con fluidi sia allo stato liquido sia allo stato gassoso. Una delle maniere più semplici per ottenere una cella di convezione allo stato liquido è quello di prendere un vaso di vetro di forma cilindrica, pieno di acqua e di riscaldare il centro della base. I movimenti delle varie particelle liquide, sono

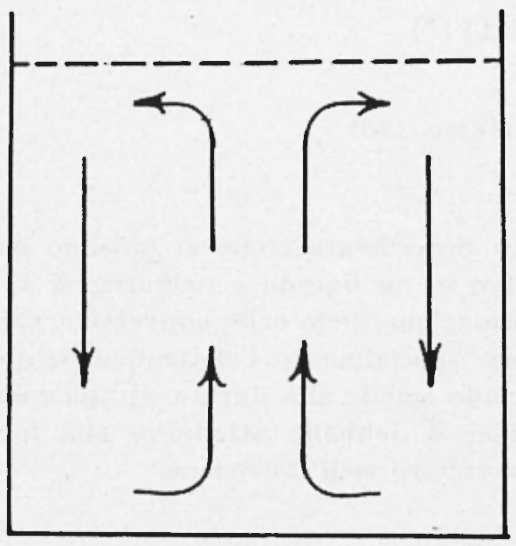

Fig. 1

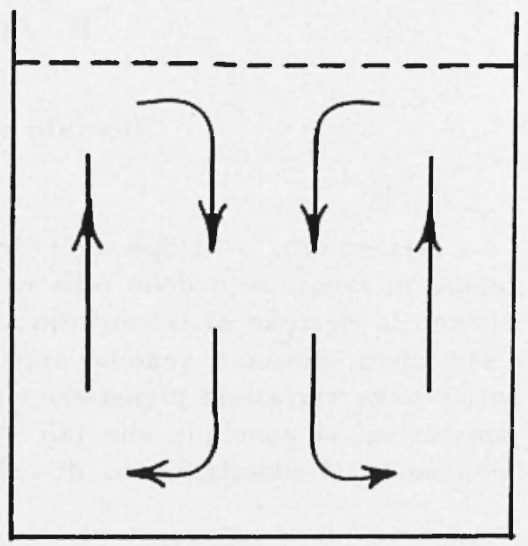

Fig. 2

mostrati dalla Fig. 1, che è una sezione del vaso ottenuta, conducendo un piano ortogonale alle due basi passante per i loro centri. Un figura simile si otterrebbe raffreddando il centro della base superiore, per es. collocando in esso un piccolo pezzo di ghiaccio. Però, in tal caso, i movimenti delle particelle avverrebbero in senso opposto: cioè nel centro della base superiore le particelle liquide si muoverebbero verso il basso, mentre alla periferia si muoverebbero verso l'alto (Fig. 2).

\section{Natura DefLA Cella convet'tiva.}

Per studiare in maniera dettagliata in laboratorio le celle convettive allo stato liquido, basta prendere un recipiente profondo circa $10 \mathrm{~cm}$ e riempirlo con un liquido molto volatile, per es. benzolo. Per rendere visibili i movimenti delle particelle liquide, si può versare nel 
medesimo recipiente un po' di porporina. L'evaporazione del liquido produce un raffreddamento cosi rapido alla sommità dello strato liquido da causare una instabilità molto accentuata. Ora si osserva che, quando l'instabiliti di un liquido inizialmente in quiete viene interrotta, il fluido si divide in un certo numero di celle prismatiche poligonali. In un liquido il movimento generalmente si compone di un moto ascendente nel centro della cella, di un moto verso i margini alla sommità, di una discesa lungo i margini esterni e di un moto verso il centro alla base della cella, come si è visto nella Fig. 1 .

Ora, nel nostro caso, appena il liquido volatile viene versato nel vassoio, esso appare molto disturbato senza mostrare forme ben definite. Se il liquido viene esposto ad una debole corrente d'aria, o se anche in aria calma non viene coperto, lo stato di disturbo violento continua per lungo tempo. Invece se il vassoio contenente il liquido volatile viene coperto con una lastrina di vetro, che non sia in contatto con gli orli del vassoio, la rapida evaporazione del liquido viene interrotta e le celle di formano immediatamente e mantengono la loro forma tipica per un tempo quasi indefinito.

Similmente negli esperimenti sopra le celle convettive poligonali in aria, esiste uno stadio iniziale, nel quale l'aria viene divisa in celle prismatiche poligonali di 4,5 , 6, lati.

Una delle maniere più semplici per realizzare celle convettive in aria è quella adoperata da Chandra a Londra nel Collegio Imperiale di Scienze e di Tecnologia, intorno al $1936\left({ }^{1}\right)$. Chandra adopero nei suoi esperimenti una scatola, la cui base consisteva in una lastra di acciaio dello spessore di $\mathrm{cm} 0,83$ e di area $\mathrm{cm}^{2} 929,03$. La piastra metallica poteva essere riscaldata in basso facendo passare la corrente elettrica attraverso un certo numero di resistenze disposte sotto la base della scatola stessa. La sommità della scatola consisteva in una grossa lastra di vetro quadrata avente un'area di $\mathrm{cm}^{2}$ 1451,61, inseritta in una cornice di legno, in modo da formare un recipiente che potesse essere riempito di acqua fredda. In tal modo la parte superiore della scatola poteva essere tenuta ad una temperatura approssimativamente costante. Una serie di sostegni di ottone serviva poi per tener la lastra di vetro a varie distanze dalla lamina metallica (da 2 a $16 \mathrm{~mm}$ ). Infine le pareti della scatola venivano chiuse per mezzo di strisce di feltro inserite tra la piastra metallica e la lastra di vetro. Tre termometri a resistenza di platino, posti due rispettivamente in vicinanza delle due lastre metalliche e di vetro e il terzo a metà strada tra le due lastre, servivano per misurare le differenze di temperatura. Infine, per mettere 
in evidenza le celle convettive, la scatola veniva riempita con fumo di sigaretta per mezzo di una pompa. Chi scrive, in collaborazione con l'ing. Renzo Fedrigo, ha realizzato una scatola simile, il cui schema si vede nella Fig. 3. Gli esperimenti eseguiti confermarono pienamente i risultati di Chandra. Si ì trovato che i movimenti del fumo variano in maniera notevole in dipendenza della profondita della scatola e della diflerenza di temperatura tra la base e la sommita (Vedi fotogratie di Chandra).

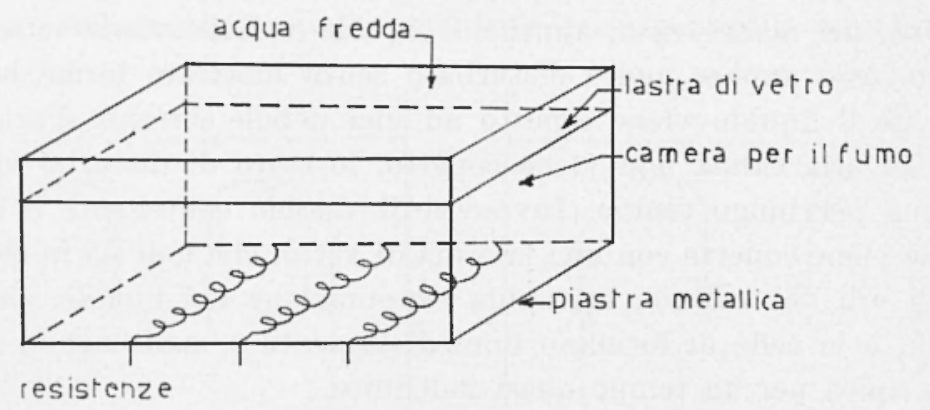

Fir. 3

Le forme ottenute più salienti, si possono classificare in 3 gruppi:

1. - Con una profondità uguale o maggiore di $10 \mathrm{~mm}$, le forme assunte dal fumo sono in parte celle prismatiche poligonali, nelle quali alla sommita il movimento avviene verso il centro, nel centro verso il basso e alla periferia verso l'alto (Fig. 2) ed in parte di coppie di "rotoli " con moto discendente lungo il confine comune. Però tali forme non rimangono fisse, giacché i rotoli si spezzano in celle prismatiche poligonali, mentre le celle prismatiche si riuniscono fra di loro per formare dei lungi rotoli in un modo apparentemente irregolare.

I diametri poi delle celle poligonali e la grandezza dei rotoli, aumentano ron l'aumentare della profondità della scatola e della differenza di temperatura tra la base e la sommità. Quando la differenza di temperatura diviene molto grande, i confini delle figure tendono a divenire ondulati.

2. - Quando la profondità della scatola è di $6 \mathrm{~mm}$ o meno, le figure assunte dal fumo non assomigliano per nulla a celle prismatiche poligonali. Peró, anche in tal caso, il fumo assume in parte la forma di coppie di rotoli. Appena viene introdotto nella scatola del fumo, 
questo si speza in strisce parallele alla direzione del moto del fumo. Dopo un breve intervallo di tempo si forma in prossimita della piastra calda,uno strato di piccolo spessore di aria biancastra, la quale si precipita verso la sommità sotto forma di piccole colome verticali.

3. - Con una profondità compresa fra $10 \mathrm{~mm}$ e $7 \mathrm{~mm}$ si osserva che, quando la differenza di temperatura tra la base e la sommità i relativamente piccola, le configurazioni del fumo sono del primo tipo, mentre con grandi differenze di temperatura sono del secondo tipo, con la sola differenza che le coppie dei rotoli, vengono, in gran parte, rimpiazzati da celle prismatiche poligonali con moto discendente al centro.

Con profondità di $6 \mathrm{~mm}$ o meno è impossibile produrre forme del primo tipo a meno che sopra la lastra di vetro non si metta aria liquida in modo che la differen\%a di temperatura tra la base e la sommita sia elevatissima.

PROVE SPERIMEN'ALI DEIAA FORMULA DI JGFFRISS.

Lord Rayleigh, nel 1.916, in ma discussione matematica sopra la rottura della stabilita in strati di fluido riscaldati dal basso, trovò che nno strato di fluido più denso alla sommità che alla base, poteva rimanere in equilibrio, finché venisse soddisfatta la condizione:

$$
\frac{\varrho_{1}-\varrho_{0}}{\varrho_{0}}-\frac{27 \pi^{1} \chi^{v}}{4 g h^{3}}
$$

dove $\varrho_{1}$ e $Q_{0}$ sono le densità del fluido rispettivamente alla sommità e alla base, $\chi$ il coefficiente di diffusione molecolare del calore, $v$ il coefficiente cinematico di viscosità, $g$ l'accelerazione della gravità e $h$ l'altezza dello strato.

Nel caso dei gas la precedente condizione puó essere sostituita con la seguente:

$$
\frac{T_{0}-T_{1}}{T}<\frac{2 \pi \pi^{4} \chi^{\nu}}{4 g h^{3}}=\frac{658 \chi^{\nu}}{g h^{3}}
$$

dove $T_{0}$ e $T$ sono le temperature rispettivamente alla base e alla sommità della scatola e $T$ la temperatura intermedia. 
Jeffrey's nel 1928 mostrò che l'ultima disuguaglianza si doveva sostituire con la seguente:

$$
\frac{T_{0}-T_{1}}{T}<\frac{1709 \gamma \nu}{g h^{3}} \text { ovvero } \frac{T_{0}-T_{1}}{\chi^{\nu} T}<\frac{1709}{g h^{3}} .
$$

Fu dimostrato sperimentalmente che lo strato di aria poteva rimanere in equilibrio con una temperatura più alta alla base che alla sommiti. Quando la piastra di base viene riscaldata, non si osserva nessun movimento nella scatola, finché la differenza di temperatura tra la base e la sommità non supera un certo valore limite, che dipende dallo spessore dello strato. Tale differenza di temperatura viene chiamata "differenza critica ". Per es. con uno spessore di $10 \mathrm{~mm}$ non si rileva nessun movimento, finché la differenza di temperatura non superi $11,4^{\circ} \mathrm{C}$ circa.

Chandra determinò sperimentalmente le differenze critiche di temperatura in funzione della profondità della scatola.

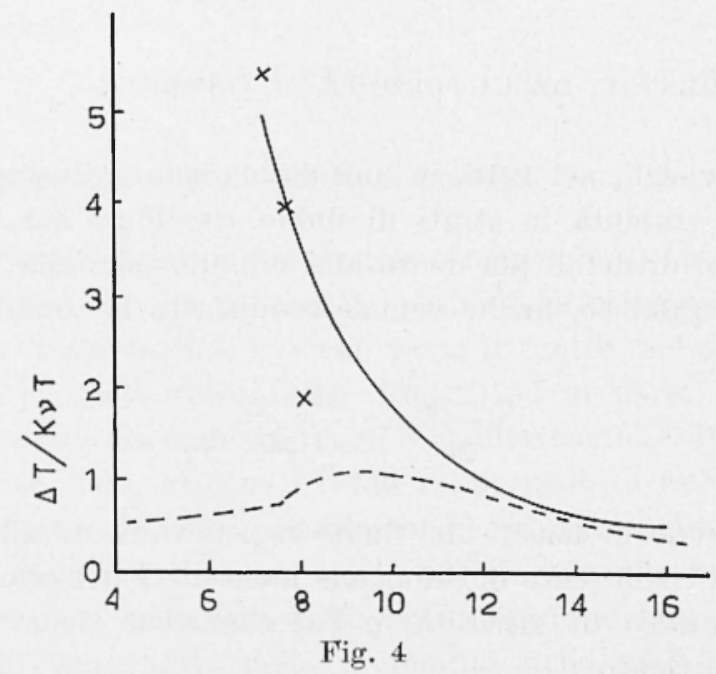

Nella Fig. 4 sulle ascisse sono riportate le profondità della scatola, mentre sulle ordinate sono riportate le differenze critiche di temperatura, divise per il prodotto $\chi \nu T$. La linea tratteggiata si è ottenuta sperimentalmente, mentre la linea continua si è costruita teoricamente per mezzo della formula di Jeffreys. Come si rileva dalla Figura, i due diagrammi sono in buon accordo con profondità superiori agli $11 \mathrm{~mm}$, sono in discordanza con profondità minori. 
APPLICAZTONI ALIATMMOSFERA TERRESTRE.

E logico che nell'atmosfera difficilmente si presentino condizioni identiche a quelle che damno origine ai fenomeni di laboratorio sopradescritti.

Però è ovvio che in certi casi possano presentarsi condizioni simili. Per es. alle $9^{\text {h }}$ del 23 Marzo 1964 lo scrivente ha osservato sopra Venezia un grande strato di nubi all'altezaa di circa 6000 metri, molto simili alle fotografie delle celle convettive ottenute da Chandra. Tale strato presentava molte figure poligonali con 4 o 5 lati. Attraverso il centro di ciascuna di esse si scorgeva il cielo sereno. Pare che esso possa essere considerato come una riproduzione in grande delle celle convettive ottenute in laboratorio. Siccome al centro di ciascuna cella si vedeva il cielo sereno, il movimento al centro doveva essere rivolto verso il basso, mentre alla periferia verso l'alto, come negli esperimenti di laboratorio. Cosi pure a Cima Sappada (a $1300 \mathrm{~m}$ ) nei primi giorni del 1960 ha osservato dei rotoli molto simili a quelli ottenuti in laboratorio (veri fotografia dell'autore). - Si fa osservare che l'apparizione di questi rotoli segnó il passaggio da bel tempo a cattivo tempo.

Inoltre le celle convettive di laboratorio possono illuminarci sopra molti movimenti dell'aria, che avvengono nell'atmosfera. Per es.:

1. - Il riscaldamento della bassa troposfera, prodotto dai raggi solari, dà origine ai cumuli, che sono frequenti nei pomeriggi estivi e ai cumuli che spesso si sviluppano quando masse di aria fredda scorrono sopra una superficie calda.

2. - 11 raffireddamento della media troposfera può causare una condizione di instabilità nell'atmosfera più bassa. I cumuli notturni, che si formano sopra gli oceani, possono essere attribuiti, almeno in parte, a tale processo.

3. - Nello sviluppo di celle convettive può avvenire la saturazione di 1110 strato d'aria instabile dal punto di vista convettivo. Un magnifico esempio di questo processo ei è dato dalle precipitazioni delle celle convettive delle regioni marittime tropicali.

Da quanto è stato detto sopra, è intuitivo che sotto certe condizioni ben determinate, le celle convettive possono formarsi nell'at- 


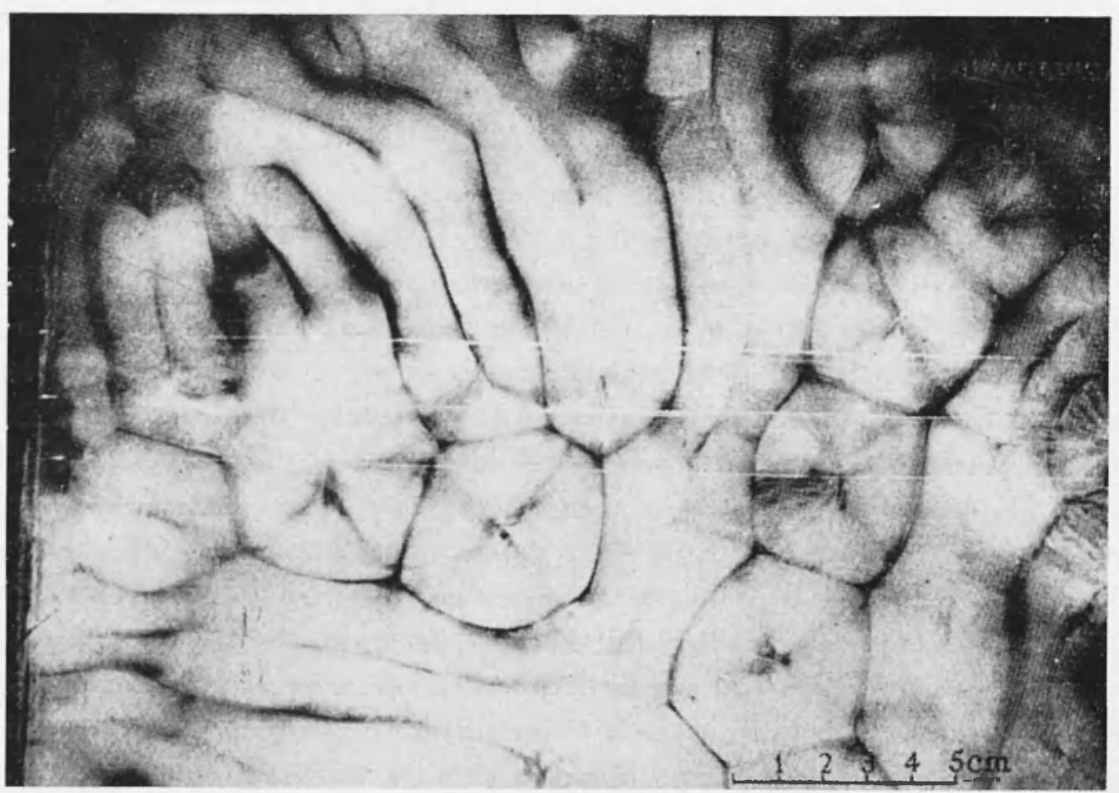

Foto 1 - Fotografia ottenuta da Chancira in laboratorio.
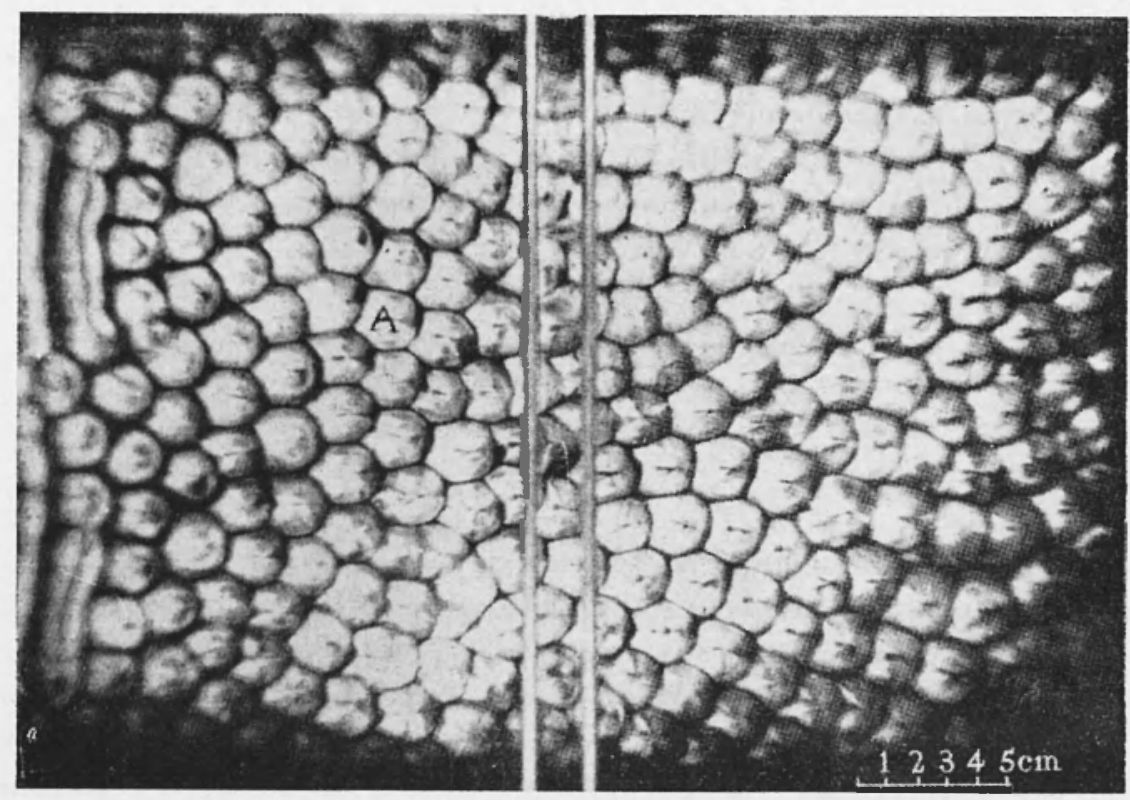

Foto 2 - Fotografia ottenuta da Chandra in laboratorio. 


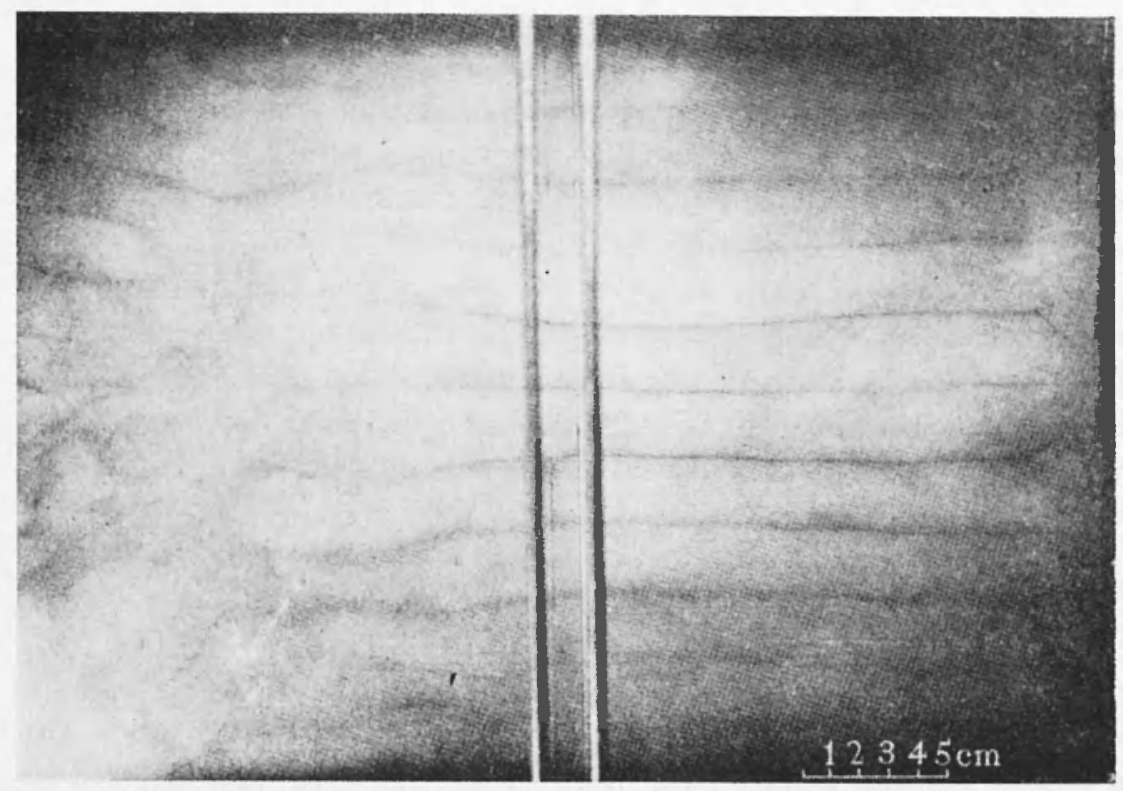

Foto 3 - Fotografia ottenuta da Chandra in laboratorio.

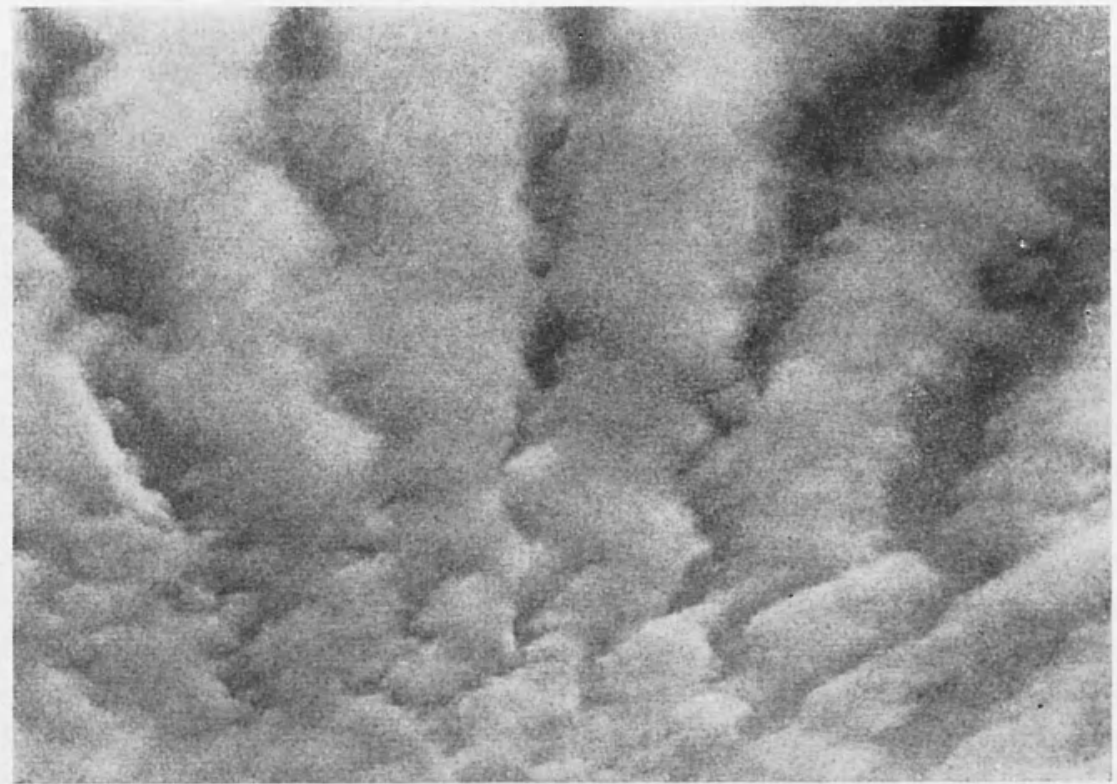

Foto 4 - Fotografia ottenuta dallautore a Cima Sappada (1300 m). 
mosfera con qualunque tempo: durante i temporali, con pioggia più o meno intensa, con cielo coperto senza pioggia, e anche con cielo sereno. Ora, specialmente i diagrammi descritti da tre strumenti, almeno a Venezia, confermano questa semplice intuizione, e precisamente i diagrammi tracciati dall'igrografo, dall'elettrometro e dal solarigrafo.

Se una massa d'aria si trova immersa in aria più fredda, verificatesi certe condizioni, essa salirà in alto. Precisamente, se indichiamo con $T_{1}$ la temperatura di tale massa d'aria e con $T$ la temperatura dell'aria circostante (con $T_{1}>T$ ), ciascuma molecola di questa massa d'aria sarà soggetta ad una accelerazione diretta verso l'alto data dalla formula:

$$
\frac{d V_{2}}{d t}=z \frac{T_{1}-T}{T}
$$

dove $V_{z}$ è la componente della velocità lungo la verticale e g l'accelerazione della gravità. Se ammettiamo che, salendo in alto tale massa d'aria si espanda adiabaticamente, è ovvio che essa sarà soggetta ad un continuo raffreddamento ed in conseguenza di ciò il vapor acqueo in essa contenuto diverrà saturo. Così hanno origine i cumuli di converione.

Secondo estesi dati sperimentati di Byers e Braham $\left(^{2}\right)$ la cir. colazione nell'interno di una cella tipica nello stadio di cumulo ci è data della Fig. 5.

In analogia con le celle temporalesche $\left(^{3}\right)$, possiamo dare uno schema dello svolgimento di una cella convettiva di bel tempo nel seguente modo:

Dapprima si forma una colonna d'aria ascendente la quale espandendosi si raffredda, perciò ad una certa altezza il vapor acqueo in essa contenuto si condenserà per formare delle nubi. Quando le gocce d'acqua così formate avranno raggiunto un peso superiore alla spinta ricevuta dalla corrente d'aria ascendente, la cella ha raggiunto il suo stadio di maturità. Tali gocce discendendo verso il basso, anche se non raggiungono il suolo, avranno la funzione di annullare, almeno in parte, la corrente d'aria ascendente. Molto probabilmente al dissolvimento della colonna d'aria ascendente concorre anche l'irruzione in essa dell'aria circostante più fredda. Cosi un po' alla volta la cella convettiva viene estinta. Per mezzo del raffreddamento, in causa dell'espansione adiabatica e del mescolamento con l'aria circostante, si potrebbe spiegare anche il dissolvimento della cella convettiva nel 
aso in eui non si ha formazione di nubi. Naturalmente alla prima cella ne può seguire una seconda e a questa una terza e cosi via, finché persistono rerte condizioni, quali per esempio, un opportuno spessore

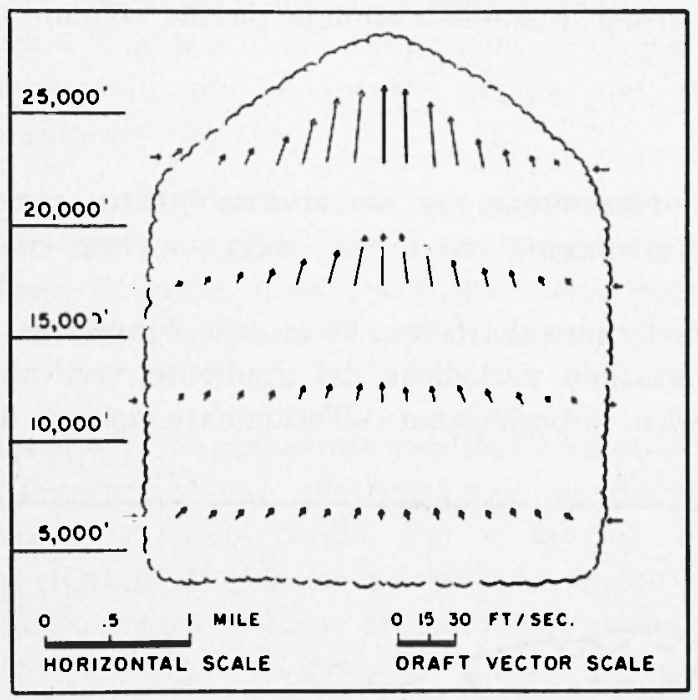

rig. 5.

dello strato d'aria in cui hamno sede i moti convettivi e la differenza di temperatura tra la base e la sommità dello strato stesso. Quanto alla durata di una cella convettiva, secondo gli elettrogrammi, essa è compresa tra 12 e 30 minuti circa. E ovvio che tale durata varii entro un intervallo di tempo piuttosto grande, giacché anch'essa dovrebbe dipendere, in gran parte, dallo spessore dello strato e dalla differenza di temperatura tra la base e la sommità del medesimo. Però la durata di una cella, secondo gli igrogrammi, ordinariamente i compresa fra i 15 e i 20 minuti circa. E ciò è naturale, gracché l'igrografo ci rivela l'esistenza di celle convettive solo con tempo relativamente bello (cielo coperto o sereno). E ovvio che quando la umidita relativa in vicinanza dello strumento è piuttosto rilevante, non può essere influenzata in maniera sensibile da fenomeni che avvengono negli strati piǹ elevati. is pure interessante il fatto che con piograia debole o moderata, frequentemente, anche gli elettrogrammi rivelino una durata compresa tra $12^{\mathrm{m}}$ e $20^{\mathrm{m}}$ circa. Con tempo buono o con pioggia moderata e calma, la vita della cella può svolgersi in maniera più regolare che con tempo pervaso da grandi disturbi, come avviene ordinariamente durante i 
temporali. Ovviamente le nubi che si formano internamente alle celle convettive, hamno la proprietà importante di renderle visibili. Questo vale sia per le nubi di grande massa, come generalmente sono i cumuli convettivi, come per le picrole nubi, p. e. per i cirro-cumuli. Quindi anche queste ultime rendono visibili le piccole colonne d'aria ascendente.

\section{VARIZIONI PERIODICHE DFI, (ARADIENTE VERTICALE DEL CAMPO ELFITTRO-ATMOSFERICO PRODO'I"IE DALIE CEILE CONVETTIVE.}

Per renderci conto del fatto che le celle convettive possono produrre delle variazioni periodiche del gradiente verticale del ampo elettroatmosferico, incominciamo coll'esaminare come si dist ribuiscono

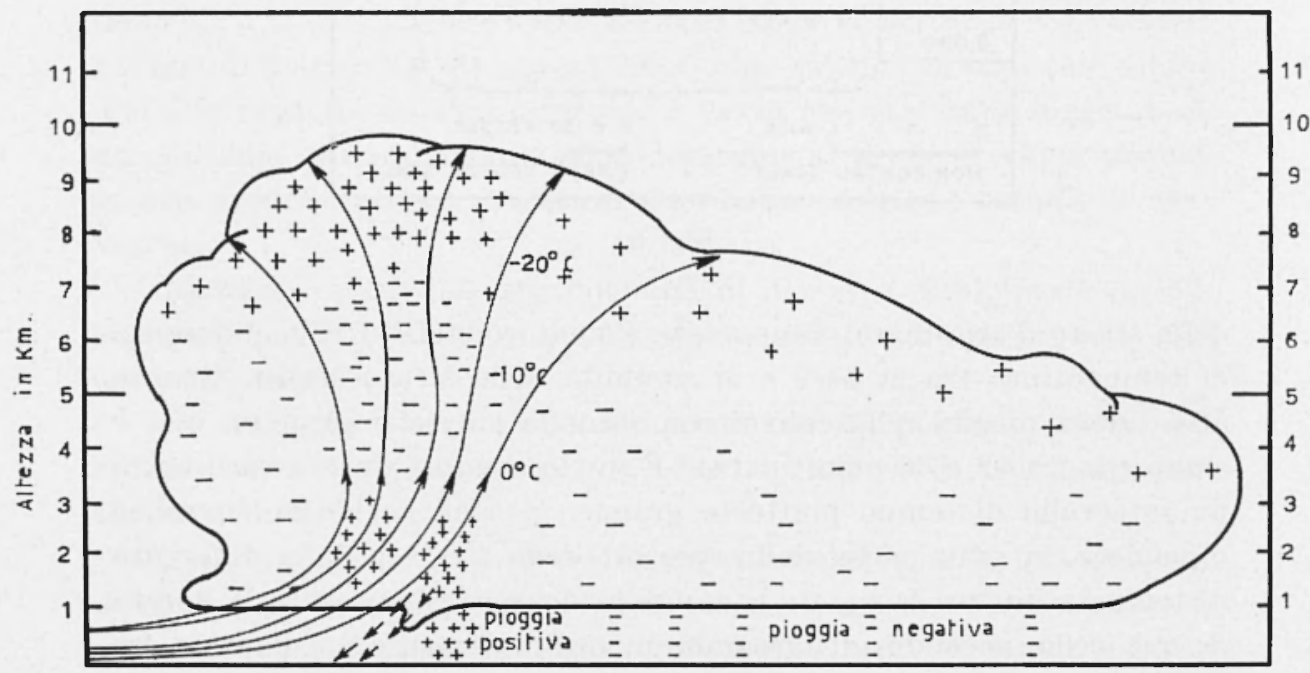

Fig. 6 .

le cariche elettriche nell'interno di una cella temporalesca (Fig. 6). Riguardo al meccanismo con cui le cariche elettriche vengono generate e separate internamente ad una nube temporalesca, ci sono ancora dei punti oscuri. Però mi sembra che una teoria sufficientemente plausibile possa fondarsi sopra le quattro scoperte sperimentali seguenti:

1. - Quando una goccia d'arqua si spezza in altre gocce più piccole, per esempio per spruzzamento, le goccioline assumono una carica 
elettrica positiva, mentre l'aria circostante rimane carica negativamente. In ciò consiste l'efletto Iénard.

2. - In recenti esperimenti di laboratorio si i scoperto che il congelamento di goccioline d'acqua è spesso accompagnato dallo scoppio dell'involucro di ghiacrio, con l'espulsione di piccoli frammenti di ghiaccio carichi positivamente, mentre rimane ma carica negativa sul resto del ghiaccio rimasto.

3. - Pure in ricerche di laboratorio si è scoperto che quando si spruzzano delle gocce soprafiuse sopra una superficie fredda per produrre uno strato di brina, questa acquista, nella maggior parte dei casi, una carica negativa, mentre l'aria circostante ne assume una positiva.

4. - Finalmente si è dimostrato sperimentamente che un chicco di grandine può venire caricato elettricamente quando viene urtato da piccoli cristalli di ghiacccio, purché essi si trovino a temperatura differente: un cristallo di ghiaccio un po' più freddo (del chicco di grandine) urtando contro il chicco di grandine rimbalza con una carica positiva, mentre sul chicco di grandine rimane una carica negativa; nel caso di un cristallo leggermente più caldo le cariche hanno segni opposti. In generale la superficie del chicco di grandine sarà un po più calda dei cristalli di ghiaccio a causa del calore latente, che si libera quando nuove goccioline gelano sul chicco stesso; quindi la grandine acquisterà una carica negativa. Il fatto che quando un involucro di ghiaccio scoppiando espella frammenti di ghiaccio carichi positivamente mentre sul resto del ghiaccio rimane una carica negativa, potrebbe dipendere da una proprietà fondamentale termoelettrica del ghiaccio, (ioè dalla diffusione preferenziale dei protoni in presenza di un gradiente di temperatura.

Quindi possiamo spiegare la generazione e la separazione delle cariche elettriche nelle celle temporalesche nel modo seguente:

Nella parte più bassa della nube, in cui la temperatura è sopra $0^{\circ} \mathrm{C}$, la colonna d'aria ascendente, urtando contro gocce d'acequa, rompe queste in grocce più piccole. Quest'ultime, per effetto Lénard. acquisteramno una carica positiva e l'aria circostante una carica negativa. Ise gocce d’acqua caricate positivamente, in causa del loro peso, rimangono alla base della nube, mentre gli ioni negativi vengono trasportati in alto. Cosi si spiegherebbe la piccola carica positiva, che ordinariamente si trova nella parte inferiore della nube. 
Nelle parti più elevate della nube, dove la temperatura è parechi gradi sotto zero, molte gocce d'acqua liquida, congelando scoppieranno, espellendo piccoli frammenti di ghiaccio carichi positivamente, mentre sul resto del chic'o di ghiacio rimarrà una carica negativa. I frammenti di ghiaccio poi, essendo leggeri, saranno portati in alto sempre dalla colonna d'aria ascendente. Invece i chicchi di ghiaccio diverranno sempre più pesanti e aumenteranno la loro carica negativa per l'urto contro goce soprafîse di ac'qua. Cosi nell'aria prossima alla brina formatasi sui chicchi di ghiaceio si troveranno altri ioni positivi, che saranno anch'essi trascinati in alto dalla colonna d'aria ascendente.

Invece i chicchi di ghiaccio, in causa del loro peso, discenderamno verso la base della nube temporalesca. Quindi, come risultato finale si avrà nell'interno della cella temporalesca una distribuzione di cariche elettriche data dallo schema della Fig. 6.

Siccome un temporale in generale si sviluppa attraverso una successione di celle, la cui vita si svolge approssimativamente nell'identica maniera (*), è ovvio che un elettrometro sitnato al disotto della nube temporalesca descriva un diagramma contenente tratti di curva molto simili tra di loro, tracciati in intervalli di tempo grosso modo eguali. Il che da molti anni è confermato dagli elettrogrammi di Venezia, i quali frequentemente, presentano curve simili ad una sinusoide.

Naturalmente quando le celle convettive non si presentano sotto l'aspetto temporalesco, i fenomeni elettrici, che avvengono internamente ad esse, sono molto meno imponenti e quindi molto meno rilevanti le variazioni del gradiente verticale del ampo elettroatmosferico al suolo. Però certe variazioni periodiche del gradiente verticale del campo elettroatmosferico, rivelate dall'elettrometro durante tempo bello, sembra che si possano spiegare ammettendo che in alto, sopra l'apparecchio, si stiano sviluppando delle celle convettive.

L'autore fa osservare che a Possagno, paese a circa $300 \mathrm{~m}$ di altitudine, in provincia di Treviso, alle falde del massiccio del Grappa, mentre si formavano sopra l'elettrometro dei cumuli convettivi, l'intensita del campo elettroatmosferico aumentava, diminuiva invece durante il loro dissolvimento. Quindi durante la formazione ed il dissolvimento di un cumulo, l'elettrometro descriveva un'increspatura piuttosto accentuata. Possiamo attribuire tale aumento dell'intensità

(*) E. J. Workman riferisce che nel Nuovo Hessico, durante lo svolgimento di un temporale, si possono sviluppare persino 12 celle, la cui vita varia da 20 a 35 minuti (?). 
del campo elettroatmosferico durante la formazione del cumulo, al trasporto convettivo verso la base della nube di carica spaziale positiva e alla carica elettrica positiva separatasi alla stessa base della nube per effetto Lénard. Durante tempo bello a Venezia, l'apparecchio descrive frequentemente anche con cielo completamente sereno, delle punte distanziate tra di loro, nel tempo, con una certa regolarita ed in buon accordo con la durata di una cella convettiva, dirette verso l'estremità del campo positivo. Mi pare che tali punte si possano spiegare mediante la formazione ed il dissolvimento di celle convettive nel seguente modo:

Durante la formazione della cella si separano alla sua base molte cariche positive, le quali, per induzione, caricano negativamente il palazzo su cui è installato l'elettrometro, e soprattutto i numerosi fulmini di cui ̀̀ fornito il palazzo stesso. Tale carica negativa attrae gli ioni positivi, che per l'effetto-elettrodo, con tempo bello, si trovano sempre in abbondanza in prossimità del suolo.

Quindi improvvisamente si avrà come la formazione di una piccola nube di ioni positivi in vicinanza della sonda dell'elettrometro, e di qui un subitaneo aumento del gradiente verticale del campo elettroatmosferico. Ma immediatamente, per l'efietto corona, uscirà dalle punte dei parafulmini una carica negativa, che dissiperà in brevissimo tempo la carica positiva improvvisamente accumulata.

Quando le celle convettive si formano e si sviluppano con cielo sereno, frequentemente le registrazioni effettuate dall'elettrometro sono identiche a quelle di bel tempo con cumuli convettivi.

Quindi possiamo concludere che anche in tal caso la distribuzione delle cariche elettriche nell'interno della cella convettiva sia identica o molto simile a quella che si presenta con nubi di bel tempo.

Prima di terminare questo argomento ci sembra del più grande interesse il fatto che una cella convettiva potrebbe dar origine ad un oscillatore atmosferico. Chiamiamo così ma bolla d'aria oscillante verticalmente nell'interno di una massa d'aria avente proprietà fisiche del tutto diverse. Infatti se immaginiamo che, per una causa qualunque, sia portata via alla colonna d'aria ascendente la sua parte inferiore, si dimostra facilmente che, in certe condizioni, essa si muoverà in direzione verticale obbedendo all'equazione:

$$
\frac{d^{2} z}{d t^{2}}+k \frac{d z}{a i}+\eta z=0 .
$$

Ovviamente noi intendiamo riferirci ad un sistema di assi ortogonali, con l'asse $z$ coincidente con la verticale passante per il bari- 
centro della bolla. Essendo la [1] un'equazione a coefficienti costanti si integra facilmente. Nell'ipotesi che $z=0$ si ha notoriamente:

$$
z=C e^{-\frac{k}{9} t}\left(e^{\frac{1 \bar{\Delta}}{9}}-e^{\left.\frac{\sqrt{\Delta}}{2} t\right)}\right.
$$

dove $Q$ '̀ luna costante arbitraria e

$$
\Delta=k^{2}-4 \eta
$$

Per noi ha particolare interesse il caso in cui $\Delta<0$, poiché allora l'integrale assume la forma:

$$
z=\frac{2 w_{0}}{1^{\prime} \Delta} e^{-\frac{k}{2}} \operatorname{sen}\left|\frac{1 \Delta}{2}\right| t
$$

Quindi la bolla comincerà a muoversi verticalmente con moto oscillatorio smorzato, essendo $\frac{k}{2}$ il coefficiente di smorzamento (*).

\section{CoNCI,USIONE.}

Da quanto si è detto sopra sembra logico dedurre che molte variazioni periodiche descritte dall'elettrometro (con periodo compreso tra 12 e 30 minuti) si debbano attribuire alla formazione e al dissolvimento di celle convettive internamente all'aria sovrastante all'apparecchio. Inoltre le variazioni periodiche del gradiente verticale del ('ampo elettro-atmosferico, segnalate dall'ettrometro durante la formazione ad il dissolvimento delle celle convettive, sembrano confermare la grande importanza che ha il meccanismo convettivo nella separazione di carica elettrica spaziale $\left({ }^{6}\right)$.

Finalmente facciamo osservare che le celle convettive presentano, al giorno d'oggi, una grande attualità, giacchè la deriva dei continenti e la presenza costante di fluidi caldi negli strati più superficiali della crosta terrestre vengono attribuite da molti geofisici a moti convettivi, che avvengono nell'interno della Terra.

(*) Per chi volesse approfondire l'argomento si consiglia la consul. tazione degli articoli contrassegnati dai numeri (3) e (5) della bibliografia. 


\section{BIBLIOGRAFLA}

(1) Cinsidra K., Instability of fluids heated from below. "Royal society London Proceeding Ser. A ", Vol. 164.

() Byers II. R., Thunderstorms, "Compendium of Meteorology", A. M. S. Boston, Mass., 1951.

$\left({ }^{3}\right)$ Janeseid, R., Ricerche sul gradiente erticale del campo elettroatmosferico "Venezia. "Atti Ist. Ven. Sc. Lett." ed. Ar. Tomo GXXII, (1963-64).

(1) Mason B. J., Charge Generation in Thunderstorm, in Conosiri s. C., Problems of Almospherie and Space Electricity. Elsevier 1965.

$\left.{ }^{5}\right)$ Nania A., Oscillatori atmos/erici e ipolesi sulla nefogenesi in aria stabile. "Rivista di Meteorologia Aeronautiea ", XXII, I, (Genn.-.Iarzo 1962).

${ }^{6}{ }^{6}$ Vonsegut B., Thunderstorm Theory, in Corositi S.C., Problems of atmospheric and space electricily. Elsevier 1965.

(7) Workuax, Thunderstorm. Electricily, in Conositr s.C., Problems of atmospheric and space electricily. Elsevier, 1965. 